\title{
SELEÇÃO DE HORÁRIOS ESCOLARES COMBINANDO VALUE-FOCUSED THINKING E FITRADEOFF ${ }^{1}$
}

\author{
Rafael Verão Françozo ${ }^{a}$ *, Fábio Henrique Noboru Abe ${ }^{a}$, Luiz Felipe de Souza Jimezez ${ }^{\mathrm{a}}$, \\ Mischel Carmen Neyra Belderrain ${ }^{b}$ \\ ${ }^{a}$ Instituto Federal de Educação, Ciência e Tecnologia de Mato Grosso do Sul - IFMS \\ Corumbá-MS, Brasil \\ ${ }^{\mathrm{b}}$ Instituto Tecnológico de Aeronáutica - ITA \\ São José dos Campos-SP, Brasil
}

Recebido 03/08/2020, aceito 23/10/2020

\begin{abstract}
RESUMO
O contexto de isolamento social provocado pela pandemia da COVID-19 suscitou demandas como um maior cuidado na elaboração dos horários em instituições e ensino. O propósito deste estudo é recomendar um agendamento de horário escolar mais eficiente e que esteja em acordo com os objetivos e valores dos decisores em uma unidade da rede Federal de ensino. Para atingir este propósito, os objetivos dos decisores foram levantados por meio da abordagem Value-Focused Thinking e um conjunto de 100 horários viáveis foi gerado por meio do software ASC Timetables. Por fim os horários foram avaliados a luz dos objetivos com o método FITradeoff. Como resultado 4 horários foram selecionados para uma análise de sensibilidade e posterior recomendação final. Os resultados permitiram selecionar uma programação de horários mais eficiente e com critérios mais claros o que contribui para geração de consenso entre os envolvidos.
\end{abstract}

Palavras-chave: Análise de decisão, Análise de stakeholders, Valores, Horários escolares.

\begin{abstract}
The context of social isolation caused by the COVID-19 pandemic has given rise as greater care in the preparation of schedules in institutions and education. The purpose of this study is to recommend a more efficient high school timetabling in accordance with the objectives and values of the decision-makers in a unit of the Federal high school. To achieve this purpose, the objectives of the decision-makers were raised through the Value-Focused Thinking approach and a set of 100 viable timetables was generated through the ASC Timetable software. Finally, the schedules were evaluated in light of the objectives using the FITradeoff method. As a result, 4 timetables were selected for a sensitivity analysis and subsequent final recommendation. The results enable to select a more efficient timetable with clearer criteria, which contributes to generate consensus among those involved.
\end{abstract}

Keywords: Decision analysis, Stakeholders analysis, Values, High school timetable.

*Autor para correspondência. E-mail: rafael.francozo@ifms.edu.br

DOI: 10.4322/PODes.2021.005

${ }^{1}$ Todos os autores assumem a responsabilidade pelo conteúdo do artigo. 


\section{Introdução}

A emergência global causada pela pandemia da COVID-19 trouxe inúmeros desafios para diversos setores produtivos, entre eles o setor educacional. Em tempos de crise, analistas e gestores possuem função ativa na elaboração de modelos que permitam atenuar as dificuldades impostas. Um problema recorrente em instituições de ensino consiste no agendamento de horários entre professores e uma turma de estudantes em local e horário determinado (Pillay, 2014). Tal problema é conhecido na literatura como High School Timetabling.

A qualidade da solução gerada para o agendamento de horários em instituições de ensino tem potencial para resultar em inúmeros benefícios (Dorneles et al., 2014). Entretanto trata-se de um problema complexo especialmente por envolver um grande conjunto de atores e restrições (Al-Yakoob e Sherali, 2015). A avaliação da solução proposta é inerente aos valores e aspirações dos atores envolvidos.

Desse modo, este estudo tem por objetivo a avaliação de horários escolares gerados em uma unidade da rede Federal de ensino por meio dos objetivos baseados nos valores dos atores envolvidos considerando o cenário de isolamento social provocado pela pandemia da COVID-19. Para alcançar o objetivo proposto, este estudo é estruturado em duas fases. Na primeira fase, objetivos e critérios são identificados e estruturados por meio do método Value-Focused Thinking (VFT) (Keeney, 1992), adicionalmente um conjunto de 100 horários são gerados por meio do software ASC Timetables. Na segunda fase os horários gerados são avaliados utilizando o método FITradeoff de seleção de Almeida et al. (2016). Tal avaliação considera os atributos relacionados com os objetivos e os critérios definidos na primeira fase.

O restante deste estudo está estruturado da seguinte maneira: as Seções 2, 3 e 4 introduzem o referencial teórico a respeito dos conceitos e métodos abordados no estudo: programação de horários em instituições de ensino, estruturação de problemas por meio do VFT e o método FITradeoff. A Seção 5 descreve os procedimentos metodológicos adotados. A Seção 6 fornece uma breve descrição do contexto da situação problemática em consideração, os resultados e a discussão da intervenção. A Seção 7 apresenta as conclusões obtidas, as limitações e direções para estudos futuros.

\section{Programação de Horários em Instituições de Ensino}

Segundo autores como Brito et al. (2012); Santos et al. (2012) o problema de High School Timetabling foi introduzido na década de 60 por (Gotlieb, 1962). Desde então, vários estudos foram desenvolvidos com foco na construção dos horários (Pillay, 2014); no agendamento e balanceamento das avaliações (Müller, 2016; Cavdur e Kose, 2016; Li et al., 2015) e da construção de currículos escolares (Hao e Benlic, 2011; Achá e Nieuwenhuis, 2014; Ceschia et al., 2014).

A construção de horários escolares envolve um conjunto de restrições denominadas como "hard" ou "soft". As restrições do tipo hard são aquelas que não podem ser violadas e são categorizadas em três grupos de restrições: agendamento, eventos e recursos (Dorneles, 2015).

Restrições de agendamento consistem nos conflitos entre recursos, horários e divisão dos eventos. Por exemplo, um professor não pode ser agendado para ministrar mais de uma aula em um mesmo período; aulas não podem ser realizadas na mesma sala em um mesmo período e; o número máximo de disciplinas que podem ser ministradas em um dia.

Restrições de eventos especificam os conflitos associados a distribuição de eventos dentro dos horários disponíveis. Por exemplo, a duração definida para evento/aula; disciplinas iguais (de um mesmo evento) precisam ser agendadas de forma consecutiva no mesmo dia. As restrições de recursos especificam os conflitos associados aos recursos (professores, ambientes de ensino, estudantes). Por exemplo, um professor não pode ser agendado para ministrar aula em um período que ele não estiver disponível (Dorneles, 2015).

Quanto maior a quantidade de professores na instituição de ensino mais difícil será encontrar uma solução viável que atenda a todas as restrições do tipo "hard", dado que consequentemente 
a quantidade de restrições de eventos e recursos também aumentam. Os microdados do Censo Escolar de 2019 indicam que, em 2019, de um total de 182.468 escolas em funcionamento no Brasil, 57.432 possuíam ao menos 60 professores em atividade (INEP, 2019). Esse dado demonstra o quão comum é o problema da construção de horários nas escolas brasileiras.

As restrições do tipo soft são aquelas que devem ser respeitadas o máximo possível mas sua violação não inviabiliza a solução. (Dorneles, 2015) sugere como restrições soft: evitar períodos ociosos para os professores. concentrar as aulas dos professores no menor número de dias possível e; alocar eventos repetidos em sequência (aulas geminadas). Entretanto restrições do tipo soft podem variar entre as instituições e de acordo com os agentes envolvidos.

As restrições soft são tratadas em uma função objetivo de minimização, de modo que cada violação em uma restrição ocasiona uma penalização no valor desta, proporcional a uma ponderação definida pelos atores. O melhor cenário é aquele no qual todas as restrições flexíveis são atendidas, sendo zero o valor da função objetivo correspondente.

A diferença nos modelos educacionais entre as instituições provoca uma grande diversidade nos tipos de problemas e consequentemente nas soluções (Post et al., 2014). A proposta deste estudo apresenta um meio para avaliação de horários escolares previamente gerados, a avaliação é feita em consonância não apenas com restrições do hard ou soft mas com os objetivos baseados nos valores dos atores envolvidos.

\section{Value-Focused Thinking}

O VFT foi introduzido por Ralph L. Keeney em 1992, descrito em seu livro: Value-Focused Thinking a Path to Creative Decisionmaking e em diversas publicações vários autores (Parnell et al., 2013). O VFT propõe um meio criativo para identificar problemas de decisão, especificar objetivos e criar alternativas. Atividades cruciais relacionadas ao processo de tomada de decisão (Keeney, 1992). Segundo Keeney (1992) a literatura relacionada é focada em abordagens que demandam objetivos, alternativas e critérios previamente definidos.

No decorrer dos anos o VFT passou a ser adotado de forma crescente em problemas de decisão complexos (Parnell et al., 2013). Estudos recentes aplicaram o VFT em áreas como: Meio ambiente e energia (Lee e Leal, 2014; Irfan, 2015; Kibira et al., 2018), Tecnologia da Informação e Comunicação (Hernández et al., 2015; Sodenkamp et al., 2016; Topcu e Mesmer, 2018), Setor educacional (Sheng et al., 2010; Bernardo et al., 2018; Parreiras et al., 2019), Defesa (Linkov et al., 2012; Scala e Pazour, 2016) entre outros (Parnell et al., 2013; Françozo et al., 2019).

A base do VFT são os valores dos decisores (Siebert e Keeney, 2015). Keeney argumenta que valores são fundamentais para abordagens baseada em Pesquisa Operacional (Keeney, 1994) e para tudo mais que deseja-se fazer (Keeney, 1996). Identificar e compreender os valores das partes interessadas é tarefa crucial em um processo de tomada de decisão, uma vez que os valores expressam o que de fato interessa na questão em estudo (Gregory e Keeney, 2017, p. 490).

Através do pensamento focado em valores busca-se, primeiramente, identificar e estruturar os objetivos para depois articular as alternativas para alcançá-los (Keeney, 1996). Segundo Keeney (1992) essa forma de pensamento se contrapõe a prática comum de focar primeiro nas alternativas disponíveis e analisar quais objetivos elas atendem ou quais os critérios para avaliá-las (Keeney, 1996). Essa prática é referida como Altenative-Focused Thinking (AFT) (Keeney, 1992, 1994, 1996).

Desde sua introdução o VFT é estreitamente relacionado a modelos quantitativos de apoio à decisão como Multiple-Attribute Value Theory (MAVT), Multiple-Attribute Utility Theory (MAUT) e Multi-Criteria Decision Analysis (MCDA). Tais modelos demandam alternativas e critérios bem definidos. Focar inicialmente nos objetivos possui benefícios tais como encontrar melhores alternativas, critérios mais eficientes e oportunidades de decisão ao permitir desvendar objetivos que inicialmente não seriam considerados (Siebert e Keeney, 2015). Demais vantagens e a função central do pensamento focado em valores são ilustradas na Figura. 1. 


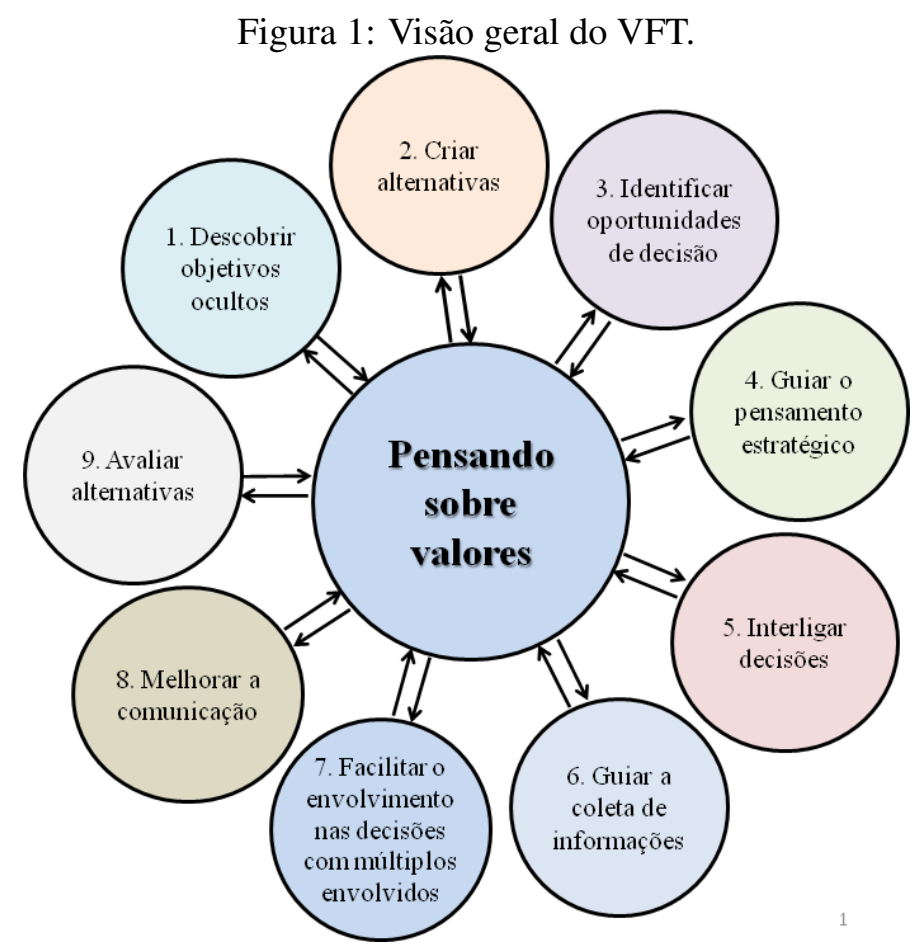

Fonte: Adaptado de Keeney (1992).

Como apresentado na Figura 1, a função principal do VFT é guiar o pensamento estratégico norteado pelos valores dos decisores. A literatura apresenta várias estruturas para aplicação do VFT sendo um dos modos mais comuns a estruturação do VFT em quatro etapas (Sheng et al., 2010; Tuhkala et al., 2017), a seguir:

1. Elaborar uma lista de valores;

2. Transformar os valores em objetivos;

3. Hierarquizar os objetivos;

4. Construir uma rede de objetivos meio-fim.

Independente do modo como o VFT é estruturado, (Keeney, 1992) argumenta que perguntas certas contribuem para a identificação de objetivos alinhados com os valores dos stakeholders. A Tabela 1 apresenta uma relação de perguntas úteis apontadas por (Keeney, 1992) para identificação dos valores e objetivos.

Tabela 1: Perguntas úteis para identificação de valores e objetivos.

\begin{tabular}{cc}
\hline Tópico & Descrição \\
\hline $\begin{array}{c}\text { 1. Desenvolver uma lista de desejos (wish } \\
\text { list) }\end{array}$ & O que você quer? O que você valoriza? O que você deveria querer? \\
\hline $\begin{array}{c}\text { 2. Identificar alternativas } \\
\text { 3. Considerar os problemas e imprevistos }\end{array}$ & O que é certo ou errado com sua organização? O que precisa ser corrigido? \\
\hline 4. Prever consequências & O que ocorreu que foi bom ou ruim? O que pode ocorrer que interessa a você? \\
\hline 5. Identificar metas, restrições e guias. & Quais são suas aspirações? Que limitações você tem? \\
\hline 6. Considerar diferentes perspectivas & Com o que o seu competidor se preocupa? Em algum tempo no futuro, o que \\
preocuparia você?
\end{tabular}




\begin{tabular}{|c|c|}
\hline Tópico & Descrição \\
\hline 7. Determinar objetivos estratégicos & $\begin{array}{l}\text { Quais são seus objetivos finais? Quais são os seus valores que são absolutamente } \\
\text { fundamentais? }\end{array}$ \\
\hline 8. Determinar objetivos gerais & $\begin{array}{c}\text { Qual objetivo você tem para clientes, empregados, acionistas e para você mesmo? } \\
\text { Que objetivos ambientais, sociais, econômicos ou de saúde e segurança são } \\
\text { importantes? }\end{array}$ \\
\hline 9. Estruturar objetivos & $\begin{array}{l}\text { Por que esse objetivo é importante? Como você pode alcança-lo? Seja específico: } \\
\text { o que você quer dizer com esse objetivo? }\end{array}$ \\
\hline 10. Quantificar objetivos & $\begin{array}{l}\text { Como você mensuraria que determinado objetivo foi atingido? Por que o objetivo } \\
\text { A é mais importante de que o objetivo B? }\end{array}$ \\
\hline
\end{tabular}

Fonte: Adaptado de Keeney (1992).

Com o auxílio das categorias de perguntas apresentadas na Tabela 1 é possível identificar os valores de um decisor e convertê-los em objetivos dentro de um determinado contexto decisório, ou seja, no ambiente onde está inserida a situação problemática.

Após a identificação dos objetivos, estes devem ser hierarquizados. A técnica conhecida como WITI Test (Why Is This Important? - Por que isso é importante?), permite identificar os objetivos meios, ou seja, aqueles que conduzem a determinado objetivo fundamental (Keeney, 1996). Com os objetivos hierarquizados é possível construir a rede de objetivos, um diagrama que apresenta o relacionamento dos objetivos meio-fim.

A partir desse ponto é possível estabelecer ações que devem ser realizadas para alcançar os objetivos. Também permite que sejam e podem ser identificadas e avaliadas as alternativas e/ou ações para tal fim. A adoção do VFT permite que seja considerada uma ampla quantidade de alternativas/ações, as quais teriam sido inicialmente desconsideradas. Dessa maneira, os decisores envolvidos podem concentrar-se sobre as consequências futuras das decisões ao considerarem as ações mais desejáveis (Alencar et al., 2017).

\section{FITradeoff}

O método FITradeoff (Flexible and Interactive Tradeoff), recentemente introduzido na literatura por de Almeida et al. (2016), foi desenvolvido como um meio para apoio à decisão por meio de informações parciais a respeito das preferências dos decisores (Frej et al., 2019). O FITradeoff opera na elicitação de Tradeoffs - um modo para estimar a importância (escala ou pesos) de um valor baseado em escolhas dos decisores (Churchman e Ackoff, 1954). Os Tradeoffs por sua vez compõe a base do Multi-Attribute Value Theory (MAVT) disseminado por Keeney e Raiffa (1976).

Em modelos aditivos como o MAVT define-se atributos desejáveis e critérios para pontuar as ações (alternativas ou objetivos por exemplo) por meio de uma função de valor aditiva que usualmente é dada por:

$$
v(x)=\sum_{i=1}^{n} k_{i} v_{i}\left(x_{i}\right)
$$

Onde $v(x)$ é o valor de desempenho global da ação $x, k_{i}$ um peso ou escala, $v_{i}$ é a função de valor do atributo único $x_{i}$ e $n$ o total de critérios a ser pontuado (de Almeida et al., 2016; Meirelles e Gomes, 2009). Considerando a avaliação de alternativas atribui-se 1 na função de valor $v_{1}$ para a alternativa com a consequência mais desejável e 0 para aquela com a consequência menos desejável. As demais alternativas recebem algum valor entre 0 e 1 . A literatura apresenta variações dessa formulação, como desconsiderar a escala $k_{i}$ ou com a agregação de pesos de múltiplos decisores (Keeney, 1992; Ferretti e Grosso, 2019).

Entretanto, a tarefa de estimar as preferências dos decisores pode ser árdua, tediosa e demandar tempo, geralmente indisponível, dos decisores além de ser fortemente sujeita a certas 
inconsistências nos julgamentos (Salo e Hämäläinen, 1992; de Almeida et al., 2016; Frej et al., 2019). O FITradeoff fornece um meio para superar tais dificuldades ao considerar a ordenação das preferências dos decisores sem necessidade elicitação de pesos e indiferenças entre elas de Almeida et al. (2016).

O FITradeoff é operado por meio um software homônimo que permite que os resultados sejam apreciados de modo interativo e intuitivo. Originalmente o FITradeoff estava limitado a escolha da melhor alternativa em um conjunto de alternativas com base na modelagem das preferências dos decisores (Frej et al., 2019). Uma versão alternativa apresentada por Frej et al. (2019) estendeu o método para classificação e ordenamento das alternativas.

Enquanto o FITradeoff é um método interativo que permite a elicitação dos pesos dos critérios por meio de informações parciais dos decisores (Holanda et al., 2017), o VFT é bastante útil para identificar os objetivos baseados nos valores dos decisores, o que ajuda a identificar mais alternativas e melhores critérios para avaliá-las (Keeney, 1992). A combinação VFT+FITradeoff é relativamente comum na literatura e pode ser encontrada em estudos recentes relacionados com fornecimento de água (da Silva Monte e Morais, 2019), seleção de projetos de sistema de fabricação (Silva et al., 2019), seleção de ferramentas de Tecnologia da Informação (Holanda et al., 2017), seleção de fornecedores (Rodrigues et al., 2020) entre outros.

\section{Procedimentos Metodológicos}

Este estudo é apoiado no framework adotado em Franco e Montibeller (2011); Ferretti e Grosso (2019); Abuabara et al. (2019) para intervenções baseadas em métodos de análise de decisão multicritério (MCDA). Neste framework a intervenção ocorre em uma fase inicial denominada fase divergente. Nesta fase, emergem incertezas a respeito dos valores e objetivos desejados assim como seus respectivos atributos. A segunda fase, denominada convergente, consiste na criação de alternativas e definição dos critérios para consequente avaliação do desempenho das alternativas. Ao final desta fase é realizada uma análise de sensibilidade e a recomendação aos decisores. $\mathrm{O}$ framework adotado é ilustrado na Figura 2.

Figura 2: Processo de Tomada de Decisão.

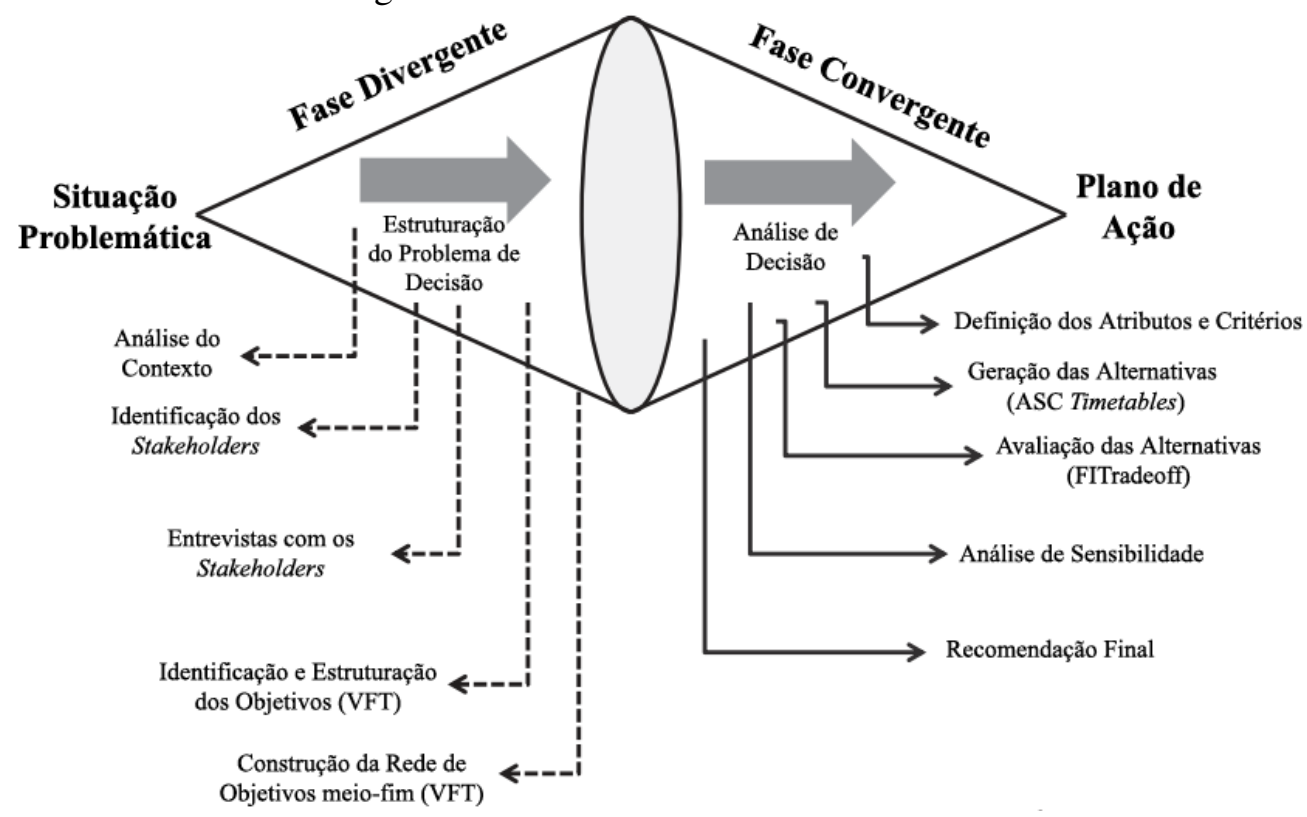

Fonte: Adaptado de Abuabara et al. (2019).

$\mathrm{Na}$ fase divergente os objetivos são identificados e estruturados seguindo os procedimentos do VFT. Entrevistas são conduzidas com os decisores visando identificar os valores envolvidos. 
Em seguida estes valores são transformados em objetivos concretos no formato Verbo+Objeto. Um valor pode dar origem a vários objetivos assim como vários valores podem ser representados em um único objetivo. Este procedimento resulta em uma "nuvem de objetivos" sem nenhuma estrutura estabelecida.

Na sequência aplica-se o teste WITI com o decisor. Para cada objetivo dentro da nuvem the é questionado: Por que isso é importante? O resultado pode apontar para outro objetivo dentro da nuvem ou para um novo objetivo. Este exercício resulta na hierarquia de objetivos na qual é possível identificar os objetivos fundamentais e os objetivos meio. A hierarquia de objetivos é representada graficamente em uma rede de objetivos meio-fim que permite uma visão holística do que é pretendido com a intervenção na situação problemática.

Com a rede de objetivos meio-fim estruturada, procede-se para a fase convergente. Nesta fase, por meio de análise da rede de objetivos meio-fim, é possível identificar os atributos dos objetivos fundamentais e os critérios para avaliação do desempenho das alternativas para tais objetivos. Neste estudo as alternativas consistem em um conjunto de 100 horários escolares aleatórios gerados por meio do software ASC Timetables.

As alternativas geradas são pontuadas segundo os atributos identificados e avaliadas por meio do método FITradeoff para escolha. Neste processo uma parte dos horários é descartada restando somente aqueles com melhor desempenho que seguem para a análise de sensibilidade. Por fim é realizada a recomendação final do horário com melhor desempenho.

\section{Resultados}

Esta seção descreve os resultados da intervenção em uma unidade da rede Federal de ensino durante o planejamento do segundo semestre letivo do ano de 2020. Ao final desta Seção é realizada uma breve discussão dos resultados obtidos com a literatura relacionada.

\subsection{Contexto da Situação Problemática}

A unidade escolar sob consideração teve as aulas presenciais suspensas e substituídas por aulas não presenciais por meio das plataformas de ensino à distância da instituição. Havia uma expectativa dos envolvidos com a retomada das aulas presenciais no segundo semestre, entretanto devido ao constante aumento no número de pessoas infectadas pela COVID-19 e esgotamento no número de leitos clínicos na região, o cenário provável é de que todo o segundo semestre letivo ocorra de forma não presencial. A preparação das aulas não presenciais demandam um esforço maior dos professores, desse modo, a adoção de um horário mais alinhado com os objetivos do decisores é fundamental para mitigar os efeitos negativos do cenário atual.

A instituição conta atualmente com um corpo docente composto de 60 professores de Ensino Básico, Técnico e Tecnológico (EBTT) divididos em especialidades distintas. Todos possuem 40 horas de carga horária semanal de trabalho. Essa carga é dividida em ensino, pesquisa e extensão. A instituição oferece cursos nas seguintes modalidades: nível técnico, superior, educação de jovens e adultos (EJA), ensino e aprendizagem a distância $(\mathrm{EaD})$ e cursos de formação inicial continuada (FIC). A infraestrutura da instituição é composta por salas administrativas, refeitório, biblioteca, quadra, anfiteatro, 6 laboratórios de informática com capacidade de até 25 computadores, 11 salas de aula com capacidade de até 40 estudantes e 6 laboratórios específicos (química, física, biologia, robótica e dois de metalurgia).

Os cursos oferecidos pela instituição são divididos de maneira semestral. Em cada início de semestre é gerado uma lista com as disciplinas que serão ministradas e cada grupo de docentes realiza a divisão por suas especialidades. Semestralmente são ofertadas 350 disciplinas com diferentes cargas horárias, totalizando 875 aulas com duração de 45 minutos cada. A Tabela 2 resume esses dados em formato de instância. 
Tabela 2: Visão Geral do Quadro de Horários.

\begin{tabular}{cccccc}
\hline Horários & Docentes & Ambientes & Estudantes & Disciplinas & Total \\
\hline 100 & 60 & 23 & 1.093 & 350 & 875 \\
\hline
\end{tabular}

Fonte: Elaborado pelos autores.

Após a distribuição das disciplinas entre os docentes uma comissão interna fica responsável por popular a base de dados do programa Applied Software Consultants Timetables, ou ASC Timetables. A instituição possui licença de uso deste software, portanto, neste estudo, optou-se por gerar horários com o ASC Timetables.

Uma vez melhor compreendido o contexto da situação problemática em consideração, é possível proceder para a aplicação do framework proposto na Seção anterior.

\subsection{Fase Divergente}

A fase divergente do estudo inicia-se pela identificação dos stakeholders. Essa identificação é realizada com auxílio da matriz de interesse $\times$ poder (Ackermann, 2011) apresentada na Figura 3.

Figura 3: Matriz Interesse $\times$ Poder.

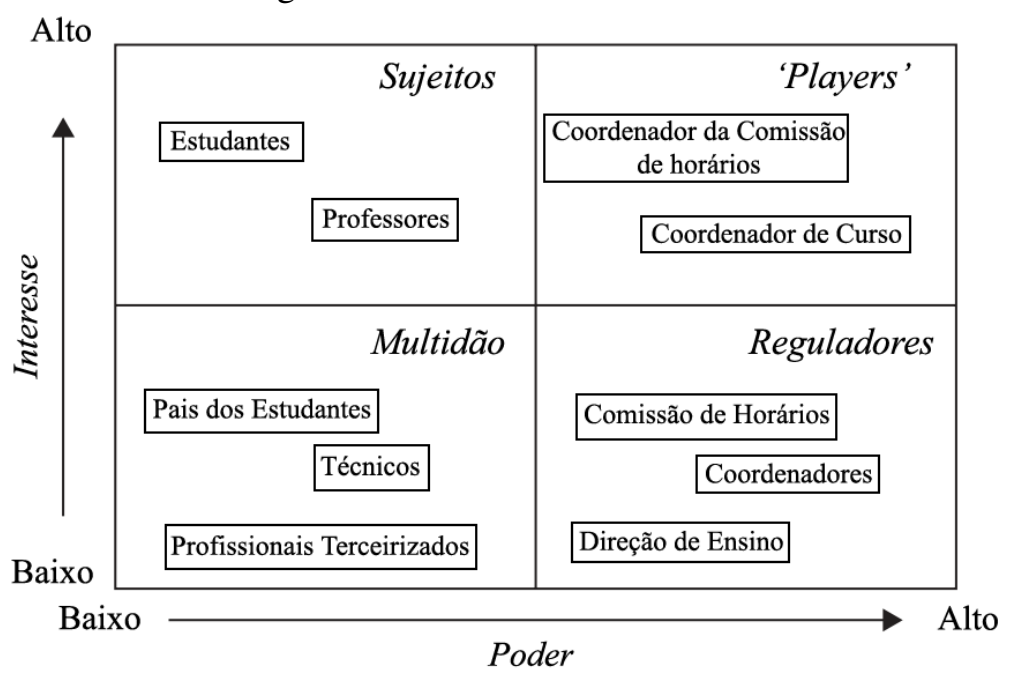

Fonte: Elaborado pelos autores.

Na matriz de interesse $\times$ poder os atores envolvidos são distribuídos de acordo com o seu grau de poder e interesse na situação problemática em consideração. A Multidão concentra aqueles com baixo grau de poder e interesse enquanto que os Players são aqueles com maior grau de poder e interesse sendo os atores mais relevantes para o estudo. Neste estudo foram classificados como players dois docentes da instituição de ensino que são os responsáveis pela geração do horário para todos os docentes da instituição.

\subsubsection{Identificação e Estruturação dos Objetivos}

Com os stakeholders devidamente identificados, procede-se para a etapa de identificação e estruturação dos objetivos. Entrevistas individuais, com duração aproximada de 45 minutos cada, foram conduzidas com os stakeholders. As 10 categorias de perguntas úteis fornecidas por Keeney e apresentadas na Tabela 1 foram utilizadas como base para definir as perguntas-guia para condução das entrevistas. As perguntas-guia são apresentadas na listagem a seguir.

1. Como você gostaria que fosse o seu horário e por que isso é importante? 
2. O que você considera que seria um horário perfeito, e o que seria um razoável?

3. O que você considera um horário péssimo?

4. Em versões anteriores o que você entende que foi bom ou ruim no horário?

5. O que você valoriza na sua atuação como docente?

Outras perguntas foram feitas ao longo das entrevistas para elucidar determinados aspectos mencionados pelos stakeholders. As entrevistas foram transcritas e validadas com os stakeholders e relevaram valores tais como: preocupações com a carga de trabalho dos docentes, desejo de atender melhor os estudantes, propiciar um ambiente favorável para o ensino, pesquisa e extensão, garantir ensino de excelência mesmo em um contexto de isolamento social, etc.

Durante o processo de validação, os valores dos stakeholders foram convertidos em objetivos concretos sendo expressos no formato Verbo+Objeto. Os objetivos identificados foram distribuídos em uma "nuvem de objetivos" apresentada na Figura 4.

Figura 4: Nuvem de Objetivos.

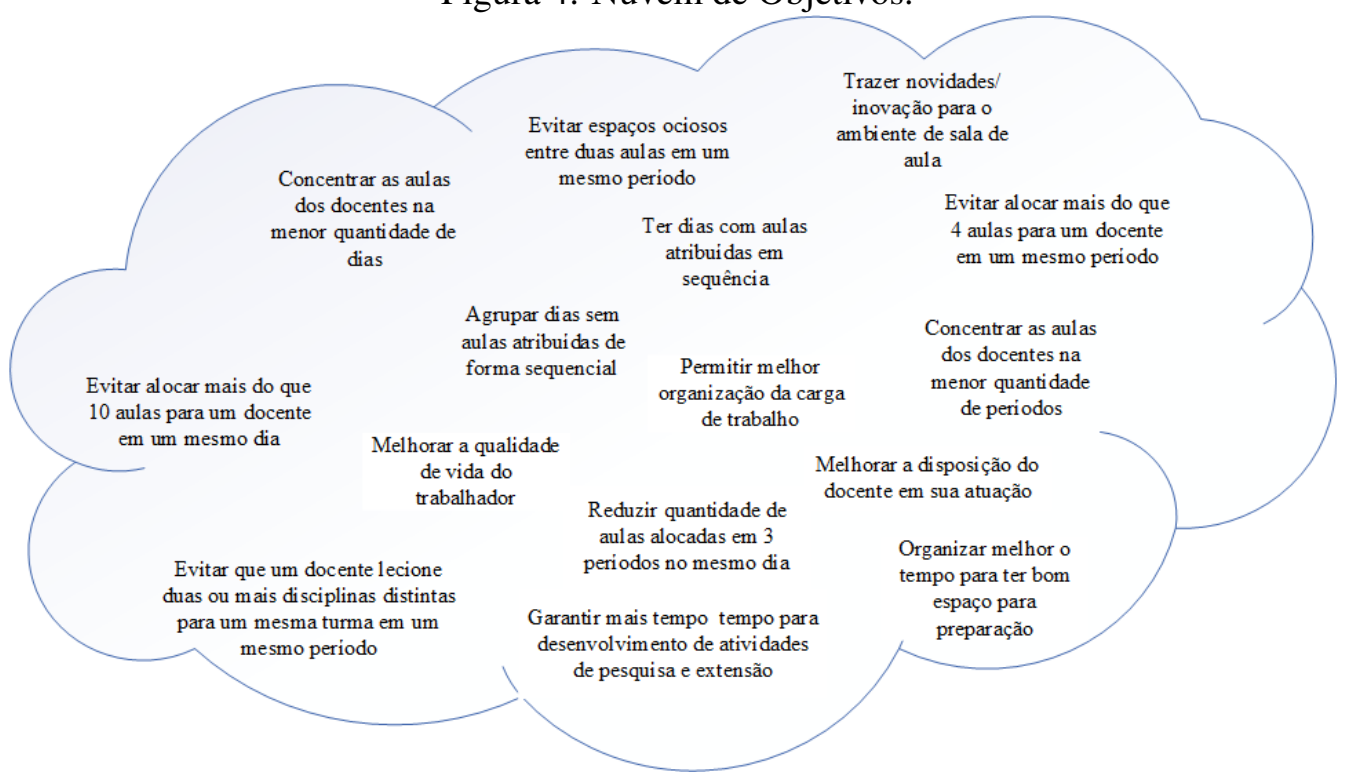

Fonte: Elaborado pelos autores.

Após a geração da nuvem de objetivos o facilitador aplicou o teste WITI com os stakeholders em grupo. Para cada objetivo na nuvem foi questionado: Por que isso é importante? As respostas apontavam para um objetivo dentro da nuvem ou indicava a geração de um novo objetivo. $O$ resultado da aplicação do teste WITI revelou a hierarquia de objetivos que por sua vez foi modelada em uma rede de objetivos-meio fim apresentada na Figura 5 
Figura 5: Rede de Objetivos Meio-fim.

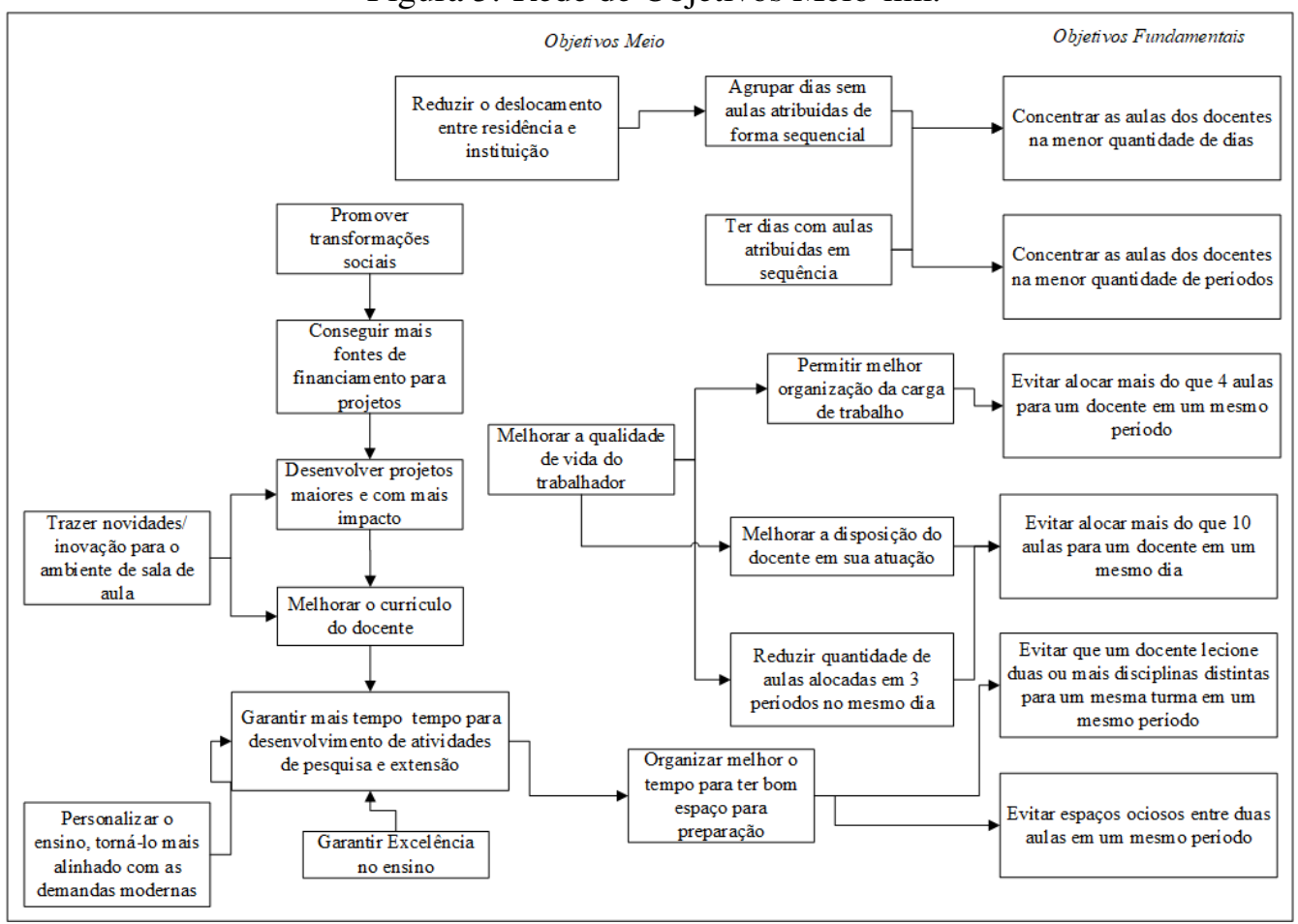

Fonte: Elaborado pelos autores.

Da rede de objetivos meio-fim apresentada na Fig. 5 destacamos os objetivos fundamentais, aqueles que, na visão dos stakeholders, são mais relevantes para a programação de horários da instituição no contexto de isolamento social. Por meio de análise dos objetivos fundamentais, os stakeholders definiram os atributos mensuráveis e a direção dos critérios para avaliação dos horários. Essas informações são apresentadas na Tabela 3.

Tabela 3: Descrição dos Objetivos Fundamentais.

\begin{tabular}{|c|c|c|c|c|}
\hline ID & Objetivo & Atributo & Direção & Rótulo \\
\hline $\mathrm{C} 1$ & $\begin{array}{l}\text { Concentrar as aulas dos docentes na menor } \\
\text { quantidade de dias }\end{array}$ & Dias com aulas alocadas & Minimizar & DAYS_ATTR \\
\hline $\mathrm{C} 2$ & $\begin{array}{l}\text { Concentrar as aulas dos docentes na menor } \\
\text { quantidade de períodos }\end{array}$ & Períodos com aulas alocadas & Minimizar & SHIFT_ATTR \\
\hline $\mathrm{C} 3$ & $\begin{array}{l}\text { Evitar alocar mais do que } 4 \text { aulas para um } \\
\text { docente em um mesmo período }\end{array}$ & $\begin{array}{l}\text { Períodos com mais do que } 4 \\
\text { aulas para um docente em um } \\
\text { mesmo período }\end{array}$ & Minimizar & M4SHIFT_ATTR \\
\hline $\mathrm{C} 4$ & $\begin{array}{l}\text { Evitar espaços ociosos entre duas aulas em } \\
\text { um mesmo período }\end{array}$ & Lacunas entre aulas & Minimizar & GAPS \\
\hline $\mathrm{C} 5$ & $\begin{array}{l}\text { Evitar alocar mais do que } 10 \text { aulas para um } \\
\text { docente em um mesmo dia }\end{array}$ & $\begin{array}{c}\text { Dias com mais que } 10 \text { aulas para } \\
\text { um mesmo docente }\end{array}$ & Minimizar & M10DAY_ATTR \\
\hline C6 & $\begin{array}{l}\text { Evitar que um docente lecione duas ou } \\
\text { mais disciplinas distintas para um mesma } \\
\text { turma em um mesmo período. }\end{array}$ & $\begin{array}{l}\text { Períodos em que um professor } \\
\text { leciona duas ou mais disciplinas } \\
\text { para uma mesma turma }\end{array}$ & Minimizar & P_FREQ \\
\hline
\end{tabular}

Fonte: Elaborado pelos autores.

Na Tabela 3 a coluna Rótulo indica o modo como o objetivo é informado no software do FITradeoff.

Outros dois objetivos inicialmente considerados foram descartados nesta etapa. O primeiro era relacionado com a redução da quantidade de docentes em simultâneo na instituição e o segundo com a redução da movimentação dos estudantes entre ambientes. Os stakeholders consideraram estes dois objetivos relevantes em um contexto de isolamento social, entretanto, como as aulas 
presenciais estão suspensas tais objetivos não teriam grande impacto neste momento. Tais objetivos podem voltar a ser considerados para avaliações futuras em um cenário de retomada das aulas presenciais.

\subsubsection{Geração e Avaliação das Alternativas}

Com os objetivos devidamente identificados e estruturados, seguiu-se a etapa de geração dos horários factíveis e aleatórios por meio do software ASC Timetables. O software ASC Timetables foi adotado uma vez que a instituição já possui licença de uso do mesmo. O ASC Timetables permite exportar os horários gerados em diversos formatos. O software ASC Timetables gera horários factíveis para o semestre letivo, mas sem considerar aspectos de otimização como os desejados neste estudo. Dessa forma, optou-se por gerar um conjunto de 100 horários para avaliá-los e escolher o mais adequado aos objetivos definidos.

Os horários gerados foram exportados no formato XML. Em seguida as informações foram processadas e armazenadas em um banco de dados. O horários foram analisados por um algoritmo próprio, desenvolvido utilizando a linguagem de programação PHP, com a finalidade de classificar, organizar e distribuir em vetores de objetos. Essa estrutura continha ainda as referências aos dados dos relacionamentos de cada objeto. Foi possível então, a partir desta estrutura, encontrar e contabilizar os atributos apresentados na Tabela 3.

A Tabela 4 apresenta a variação entre o melhor e o pior desempenho de cada horário gerado em cada critério.

Tabela 4: Variação de desempenho das alternativas em cada critério.

\begin{tabular}{cccccc}
\hline DAYS_ATTR & SHIFT_ATTR & M4SHIFT_ATTR & GAPS & M10DAY_ATTR & P_FREQ \\
\hline $225-248$ & $384-417$ & $11-32$ & $39-65$ & $0-5$ & $4-22$ \\
\hline \multicolumn{5}{c}{ Fonte: Elaborado pelos autores. }
\end{tabular}

Cada horário gerado representa o agendamento semanal das atividades. Tomando como exemplo o critério DAYS_ATTR o horário com melhor desempenho atribui um total de 225 dias com aulas considerando todos os 57 docentes o que resulta em uma média de 3,95 dias com aulas atribuídas na semana por docente. Na prática seria possível concentrar as aulas de cada docente em no máximo 4 dias da semana. Já o horário com pior desempenho para o critério DAYS_ATTR atribui o total de 248 dias, uma média de 4,35 aulas atribuídas na semana por docente. Esse desempenho implica que os docentes terão aulas em 5 dias da semana. A diferença entre o melhor e o pior desempenho é de 23 dias.

A redução na quantidade de dias com aulas alocadas permite, de acordo com a rede de objetivos, que os docentes possam organizar melhor sua carga de trabalho e reduzir possíveis deslocamentos, algo fundamental em um contexto de isolamento social. A mesma análise pode ser feita com relação aos demais critérios apresentados na Tabela 4.

\subsection{Fase Convergente}

A segunda fase do estudo é a fase convergente na qual as alternativas são avaliadas seguindo os objetivos e os critérios definidos em consenso pelos stakeholders. A avaliação das alternativas foi feita utilizando o método FITradeoff para escolha (de Almeida et al., 2016). A última atividade antes da avaliação foi definir a ordem de preferências entre os objetivos pelos stakeholders. Cada stakeholder definiu seu ordenamento preferencial destacado nos itens 1 e 2 da listagem a seguir. $\mathrm{O}$ ordenamento apresentado no item 3 foi definido em conjunto pelos stakeholders.

\footnotetext{
1. SHIFT_ATTR $>$ M10DAY_ATTR $>$ P_FREQ $>$ GAPS $>$ DAYS_ATTR $>$ M4SHIFT_ATTR

2. DAYS_ATTR $>$ SHIFT_ATTR $>$ M4SHIFT_ATTR $>$ M10DAY_ATTR $>$ GAPS $>$ P_FREQ
} 
3. DAYS_ATTR $>$ SHIFT_ATTR $>$ P_FREQ $>$ GAPS $>$ M10DAY_ATTR $>$ M4SHIFT_ATTR

Foram feitas simulações no FITradeoff seguindo os 3 ordenamentos apresentados. Durante o processo de elicitação, o FITradeoff questiona qual consequência é preferível em algumas iterações, em todas as 3 simulações foi escolhido o critério correspondente ao objetivo preferencial. Durante o primeiro ordenamento, os horários 52 e 54 tivera o melhor desempenho; para o segundo ordenamento os horários 54 e 95 e para o terceiro ordenamento os horários 54, 60 e 95 . A presença do horário 54 nas 3 simulações sugere que é um potencial candidato a ser o horário escolhido.

A Tabela 5 apresenta a pontuação dos horários selecionados nos critérios definidos.

Tabela 5: Pontuação dos Horários Selecionados.

\begin{tabular}{ccccccc}
\hline ID & DAYS_ATTR & SHIFT_ATTR & M4SHIFT_ATTR & GAPS & M10DAY_ATTR & P_FREQ \\
\hline 52 & 234 & 384 & 24 & 61 & 4 & 18 \\
54 & 227 & 384 & 32 & 61 & 0 & 13 \\
60 & 227 & 392 & 25 & 54 & 0 & 13 \\
95 & 225 & 390 & 24 & 51 & 3 & 21 \\
\hline
\end{tabular}

Fonte: Elaborado pelos autores.

Os resultados apresentados na Tabela 5 podem ser usados para apoiar um debate entre os stakeholders para escolha do horário final. Entretanto é possível refinar os resultados por meio de uma análise de sensibilidade. Embora o software do FITradeoff possua um recurso para esta finalidade, a sua adoção, mesmo com variações pequenas, resultou em um leque muito grande horários indesejados. Por essa razão optou-se por avaliar os horários normalizando os valores em cada coluna da Tabela 5 adotando o seguinte cálculo:

$$
n_{i j}=\frac{v_{i j}}{\sum_{i=1}^{n} v_{i j}}, \quad j=\{1,2,3,4,5,6\}
$$

Onde $n_{i j}$ é o valor na linha $i$ e na coluna $j$ normalizado resultante da divisão do valor atual $v_{i j}$, conforme apresentado na Tabela 5 , pelo somatório de todos os valores na coluna correspondente. A Tabela 6 apresenta os resultados deste processo.

Tabela 6: Resultados normalizados.

\begin{tabular}{ccccccc}
\hline ID & DAYS_ATTR & SHIFT_ATTR & M4SHIFT_ATTR & GAPS & M10DAY_ATTR & P_FREQ \\
\hline 52 & 0,256 & 0,248 & 0,229 & 0,269 & 0,571 & 0,277 \\
54 & 0,249 & 0,248 & 0,305 & 0,269 & 0,000 & 0,200 \\
60 & 0,249 & 0,253 & 0,238 & 0,238 & 0,000 & 0,200 \\
95 & 0,246 & 0,252 & 0,229 & 0,225 & 0,429 & 0,323 \\
\hline
\end{tabular}

Fonte: Elaborado pelos autores.

Os resultados normalizados apresentados na Tabela 6 permitem a visualização dos dados em uma mesma escala o que facilita a comparação entre o desempenho dos horários nos vários critérios. Os resultados da Tabela 6 podem ser melhor visualizados no gráfico apresentado na Figura 6 
Figura 6: Gráfico com os resultados normalizados.

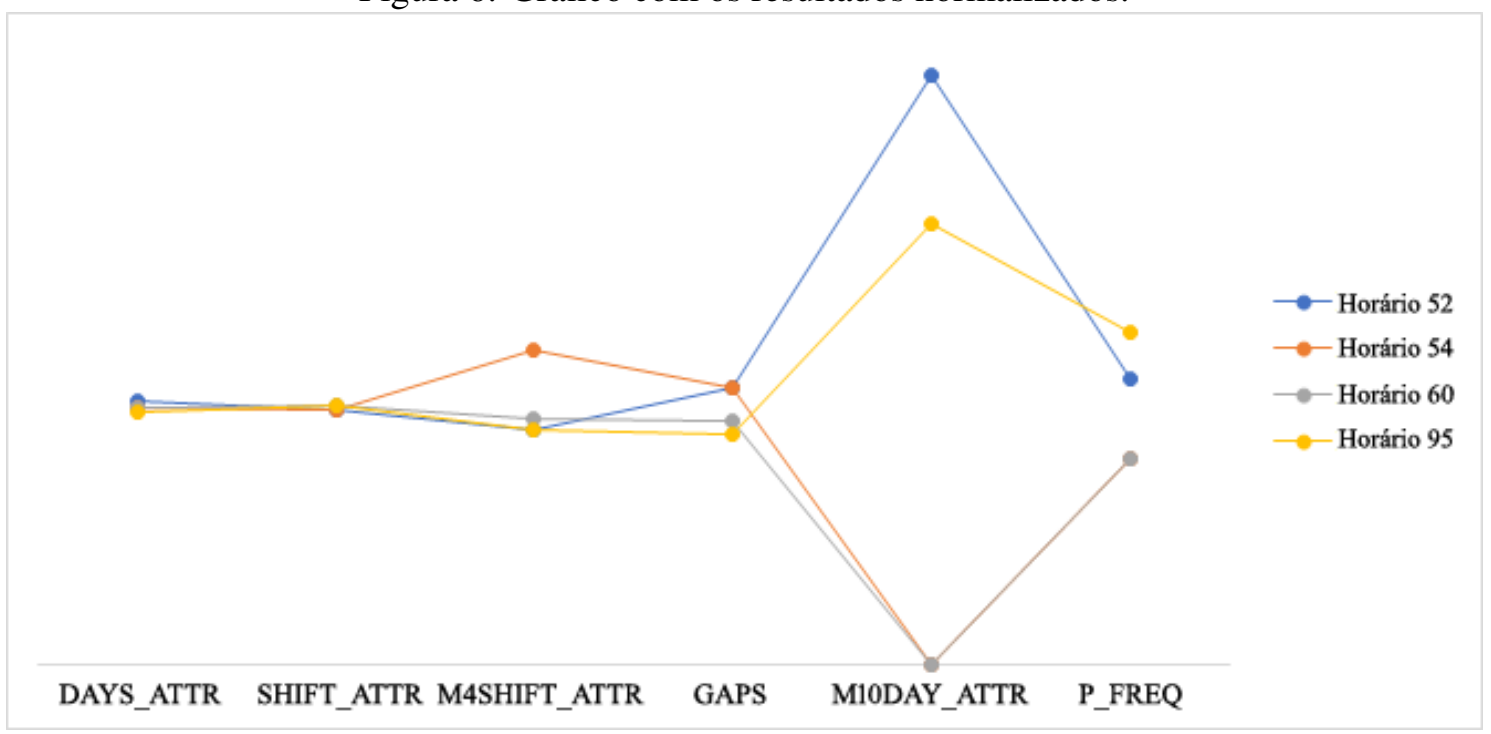

Fonte: Elaborado pelos autores.

Pela análise do gráfico apresentado na Fig. 6 é possível perceber não há variações relevantes para os critérios DAYS_ATTR; SHIFT_ATTR e GAPS o que indica que a recomendação final será feita em função dos demais critérios. Para o critério M4SHIFT_ATTR o horário 54 possui o pior desempenho, para o critério M10DAY_ATTR o pior desempenho é no horário 52 e para o critério P_FREQ o pior desempenho é do horário 95. Dessa forma a recomendação final do facilitador e em consenso entre os decisores é para o horário restante, com a id 60.

\subsection{Discussão}

O recente estudo de revisão sobre métodos para resolução de agendamento de horários escolares fornecido por Tan et al. (2021) apresenta estudos em 14 categorias de métodos como: Programação Inteira (Sørensen e Dahms, 2014; Kristiansen et al., 2015), Busca Local e Busca Tabu (Saviniec et al., 2018; Minh et al., 2010), Algoritmos Evolucionários (Andrade et al., 2019; Dutta et al., 2020; Yuan et al., 2020), Hyper-Heuristicas (Ahmed et al., 2015) e Matheurísticas (Dorneles et al., 2014; Fonseca et al., 2016), entre outros. Os resultados do estudo de revisão de Tan et al. (2021) vão na mesma direção da revisão realizada por Pillay (2014) e apontam avanços relevantes para indústria de softwares tais como o ASC Timetables.

A organização das escolas ao redor do mundo é diferente e, consequentemente, os problemas de geração de horários também são (Post et al., 2014). Desse modo a avaliação da qualidade das soluções é avaliada por meio da resolução de instâncias pré-definidas e disponível em formato XML (eXtended Markup Language) conhecidas como instâncias XHSTT ${ }^{1}$ (XML High School TimeTabling) (Post et al., 2012, 2014).

Embora os avanços nos algoritmos contribuam para encontrar soluções alternativas que satisfaçam a maior quantidade possível das restrições definidas, o julgamento humano ainda é indispensável para identificar os objetivos pretendidos e assim definir o conjunto de critérios relacionadas. Este estudo focou-se na avaliação de soluções alternativas geradas por meio de um software, considerando os objetivos e critérios dos stakeholders da instituição.

\footnotetext{
${ }^{1}$ Disponível em: https://www.utwente.nl/en/eemcs/dmmp/hstt/archives/
} 


\section{Conclusões}

O cenário de isolamento social provocado pela pandemia da COVID-19 apresentou-se como verdadeiramente desafiador para instituições de ensino forçando-as a redesenhar os processos estabelecidos em meio a incertezas e conflitos de interesses. No entanto, como em todo cenário desafiador, emergem oportunidades de mudanças. A adoção de novas práticas visando mitigar efeitos negativos sobre um processo em um determinado período tem potencial para melhorar sua eficiência em outros cenários.

Nesse contexto está inserida a instituição de ensino, foco deste estudo. A substituição das aulas presenciais por aulas não presenciais, demandou dos docentes novas formas de preparar e ministrar suas aulas. Demandou também dos gestores novas formas de organização das atividades pedagógicas de modo a reduzir os impactos das mudanças sobre docentes e estudantes com o objetivo de minimizar o inevitável prejuízo curricular.

Compreende-se que uma ação específica pode ter um impacto pequeno na contribuição deste objetivo, entretanto, um conjunto de ações tem potencial significativo de impactar positivamente no atingimento do objetivo citado. A programação de horários é uma atividade envolvida em conflitos de interesses, com múltiplos atores e divergências significativas. Tal atividade não era tratada de forma analítica no planejamento pedagógico da instituição.

O desenvolvimento deste estudo demonstrou que é possível desenvolver um planejamento de horários de modo racional afim de estar alinhado com os objetivos baseados nos valores dos decisores. De um conjunto de 100 horários avaliados, verificou-se 4 alinhados com as preferências dos decisores. O horário recomendado é um dos que possuem a maior quantidade de dias e períodos sem aulas alocadas para o conjunto de docentes e nenhum docente teve 10 ou mais aulas alocadas em um mesmo dia. O horário gerado permite que os docentes possam ter mais períodos sem aulas alocadas o que contribui para que possam planejar melhor as atividades pedagógicas.

Este estudo possui algumas limitações importantes. Primeiro a metodologia adotada foi para avaliação de horários gerados por meio de um software proprietário, ou seja, as alternativas geradas são dependentes deste software. Essa solução foi adotada em virtude do curto prazo para o planejamento dos horários para o segundo semestre do ano letivo (2020) o que inviabilizou a elaboração de outras soluções.

Outra restrição está relacionada com o fato de serem considerados somente 2 atores no processo decisório. A instituição conta com uma comissão para elaboração de horários que não pôde ser considerada neste estudo pelos mesmos motivos indicados para a limitação anterior. Tal limitação foi contornada selecionando decisores mais relevantes por meio da ferramenta de análise de stakeholders apresentada na seção de resultados.

Tais limitações não são impossíveis de serem superadas, de modo que a primeira sugestão para estudos futuros é considerar o desenvolvimento de um modelo que permita gerar horários já alinhados com os objetivos elicitados. A outra sugestão é relacionada ao envolvimento de um grupo maior de atores para identificação e estruturação de um volume maior de objetivos.

O certo é que a construção de horários na instituição antes relegada a segundo plano no planejamento pedagógico e focada em questões pouco relevantes, passa agora a ser apreciada como importante instrumento para alcançar a excelência no ensino dentro da instituição.

\section{Agradecimentos.}

Este estudo foi parcialmente apoiado pelo Conselho Nacional de Desenvolvimento Científico e Tecnológico - CNPq.

\section{Referências}

Abuabara, L., Paucar-Caceres, A., e Burrowes-Cromwell, T. Consumers' values and behaviour in the Brazilian coffee-in-capsules market: promoting circular economy. International Journal of 
Production Research, v. 57, n. 23, p. 7269-7288, 2019.

Achá, R. A. e Nieuwenhuis, R. Curriculum-based course timetabling with SAT and MaxSAT. Annals of Operations Research, v. 218, n. 1, p. 71-91, 2014.

Ackermann, F. Getting "messy" with problems: the challenges of teaching "Soft" OR. INFORMS Transactions on Education, v. 12, n. 1, p. 55-64, 2011.

Ahmed, L. N., Özcan, E., e Kheiri, A. Solving high school timetabling problems worldwide using selection hyper-heuristics. Expert Systems with Applications, v. 42, n. 13, p. 5463-5471, 2015. ISSN 0957-4174.

Al-Yakoob, S. M. e Sherali, H. D. Mathematical models and algorithms for a high school timetabling problem. Computers \& Operations Research, v. 61, p. 56-68, 2015.

Alencar, M. H., Priori JR, L., e Alencar, L. H. Structuring objectives based on Value-Focused Thinking methodology: Creating alternatives for sustainability in the built environment. Journal of Cleaner Production, v. 156, p. 62-73, 2017.

Andrade, C. E., Silva, T., e Pessoa, L. S. Minimizing flowtime in a flowshop scheduling problem with a biased random-key genetic algorithm. Expert Systems with Applications, v. 128, p. 67-80, 2019.

Bernardo, H., Gaspar, A., e Henggeler Antunes, C. A combined Value Focused Thinking-soft systems methodology approach to structure decision support for energy performance assessment of school buildings. Sustainability, v. 10, n. 7, p. 2295, 2018.

Brito, S. S., Fonseca, G. H., Toffolo, T. A., Santos, H. G., e Souza, M. J. A SA-VNS approach for the high school timetabling problem. Electronic Notes in Discrete Mathematics, v. 39, p. 169-176, 2012.

Cavdur, F. e Kose, M. A fuzzy logic and binary-goal programming-based approach for solving the exam timetabling problem to create a balanced-exam schedule. International Journal of Fuzzy Systems, v. 18, n. 1, p. 119-129, 2016.

Ceschia, S., Di Gaspero, L., e Schaerf, A. The generalized balanced academic curriculum problem with heterogeneous classes. Annals of Operations Research, v. 218, n. 1, p. 147-163, 2014.

Churchman, C. W. e Ackoff, R. L. An approximate measure of value. Journal of the Operations Research Society of America, v. 2, n. 2, p. 172-187, 1954.

da Silva Monte, M. B. e Morais, D. C. A decision model for identifying and solving problems in an urban water supply system. Water Resources Management, v. 33, n. 14, p. 4835-4848, 2019.

de Almeida, A. T., de Almeida, J. A., Costa, A. P. C. S., e de Almeida-Filho, A. T. A new method for elicitation of criteria weights in additive models: Flexible and interactive tradeoff. European Journal of Operational Research, v. 250, n. 1, p. 179-191, 2016.

Dorneles, Á. P., de Araújo, O. C., e Buriol, L. S. A fix-and-optimize heuristic for the high school timetabling problem. Computers \& Operations Research, v. 52, p. 29-38, 2014.

Dorneles, Á. P. A matheuristic approach for solving the high school timetabling problem. $149 \mathrm{f}$. Tese de Doutorado. , Universidade Federal do Rio Grande do Sul, Porto Alegre-RS, 2015.

Dutta, D., Sil, J., e Dutta, P. A bi-phased multi-objective genetic algorithm based classifier. Expert Systems with Applications, v. 146, p. 113163, 2020. 
Ferretti, V. e Grosso, R. Designing successful urban regeneration strategies through a behavioral decision aiding approach. Cities, v. 95, p. 102386, 2019.

Fonseca, G. H., Santos, H. G., e Carrano, E. G. Integrating matheuristics and metaheuristics for timetabling. Computers \& Operations Research, v. 74, p. 108-117, 2016.

Franco, L. A. e Montibeller, G. Problem structuring for multicriteria decision analysis interventions. In: Wiley Encyclopedia of Operations Research and Management Science. : , 2011.

Françozo, R. V., Belderrain, M. C. N., Bergiante, N. C. R., Pacheco, B. C. S., e Piratelli, C. L. Value-Focused Thinking na prática: análise do desenvolvimento e aplicações no período (2010-2018). In: Anais do LI Simpósio Brasileiro de Pesquisa Operacional. Limeira, SP. Galoá, 2019.

Frej, E. A., de Almeida, A. T., e Costa, A. P. C. S. Using data visualization for ranking alternatives with partial information and interactive tradeoff elicitation. Operational Research, v. 19, n. 4, p. 909-931, 2019.

Gotlieb, C. The construction of class-teacher time-tables. Communications of the ACM, v. 5, n. 6 , p. 312-313, 1962.

Gregory, R. e Keeney, R. L. A practical approach to address uncertainty in stakeholder deliberations. Risk Analysis, v. 37, n. 3, p. 487-501, 2017.

Hao, J.-K. e Benlic, U. Lower bounds for the itc-2007 curriculum-based course timetabling problem. European Journal of Operational Research, v. 212, n. 3, p. 464-472, 2011.

Hernández, M. L. C., Pantoja, E. E. P., e Reyes, B. C. G. A framework for the optimal multicriteria design of networks of small hydro turbines in polluted streams. International Journal of Multicriteria Decision Making, v. 5, n. 1-2, p. 109-128, 2015.

Holanda, N. E. d. C. et al. Modelo para seleção de uma ferramenta de TI com foco em produtividade: uma aplicação baseada no VFT e FITradeoff. . Dissertação de Mestrado. Centro Acadêmico do Agreste, Universidade Federal de Pernambuco, Cauraru-PE, , 2017.

INEP. Instituto Nacional de Estudos e Pesquisas Educacionais Anísio Teixeira. Microdados do censo escolar 2019. Disponível em: http://inep.gov.br/microdados. Acesso em: 08 Out. 2020, v. , 2019.

Irfan, S. M. A methodology for modelling energy use in the agriculture sector for the Pakistan integrated energy model. International Journal of Intelligent Enterprise, v. 3, n. 1, p. 19-37, 2015.

Keeney, R. e Raiffa, H. Decisions with Multiple Consequences: Preferences and Value Tradeoffs. New York, NY: John Wiley \& Sons, 1976.

Keeney, R. L. Value-Focused Thinking. Massachusetts: Harvard University Press, 1992.

Keeney, R. L. Using values in operations research. Operations Research, v. 42, n. 5, p. 793-813, 1994.

Keeney, R. L. Value-focused thinking: Identifying decision opportunities and creating alternatives. European Journal of Operational Research, v. 92, n. 3, p. 537-549, 1996.

Kibira, D., Brundage, M. P., Feng, S., e Morris, K. C. Procedure for selecting key performance indicators for sustainable manufacturing. Journal of Manufacturing Science and Engineering, v. 140, n. 1, p. 011005, 2018. 
Kristiansen, S., Sørensen, M., e Stidsen, T. R. Integer programming for the generalized high school timetabling problem. Journal of Scheduling, v. 18, n. 4, p. 377-392, 2015.

Lee, N. e Leal, V. A review of energy planning practices of members of the Economic Community of West African States. Renewable and Sustainable Energy Reviews, v. 31, p. 202-220, 2014.

Li, J., Bai, R., Shen, Y., e Qu, R. Search with evolutionary ruin and stochastic rebuild: A theoretic framework and a case study on exam timetabling. European Journal of Operational Research, v. 242, n. 3, p. 798-806, 2015.

Linkov, I., Valverde, H. R. L. J., Bates, M. E., Trump, B., Friedman, D., Evans, J., e Keisler, J. Civilian response corps force review: the application of multi-criteria decision analysis to prioritize skills required for future diplomatic missions. Journal of Multi-Criteria Decision Analysis, v. 19, n. 3-4, p. 155-168, 2012.

Meirelles, C. L. d. A. e Gomes, L. F. A. M. O apoio multicritério à decisão como instrumento de gestão do conhecimento: uma aplicação à indústria de refino de petróleo. Pesquisa Operacional, v. 29, n. 2 , p. $451-470,2009$.

Minh, K. N. T. T., Thanh, N. D. T., Trang, K. T., e Hue, N. T. T. Using tabu search for solving a high school timetabling problem. In: Advances in Intelligent Information and Database Systemsp. 305-313. : Springer, 2010.

Müller, T. Real-life examination timetabling. Journal of Scheduling, v. 19, n. 3, p. 257-270, 2016.

Parnell, G. S. et al. Invited review-survey of value-focused thinking: applications, research developments and areas for future research. Journal of Multi-Criteria Decision Analysis, v. 20, n. 1-2, p. 49-60, 2013.

Parreiras, R., Kokshenev, I., Carvalho, M., Willer, A., Dellezzopolles, C., Nacif, D., et al. A flexible multicriteria decision-making methodology to support the strategic management of Science, Technology and Innovation research funding programs. European Journal of Operational Research, v. 272, n. 2, p. 725-739, 2019.

Pillay, N. A survey of school timetabling research. Annals of Operations Research, v. 218, n. 1, p. 261-293, 2014.

Post, G., Ahmadi, S., Daskalaki, S., Kingston, J. H., Kyngas, J., Nurmi, C., et al. An XML format for benchmarks in high school timetabling. Annals of Operations Research, v. 194, n. 1, p. 385-397, 2012.

Post, G., Kingston, J. H., Ahmadi, S., Daskalaki, S., Gogos, C., Kyngas, J., Nurmi, C., Musliu, N., Pillay, N., Santos, H., et al. XHSTT: an XML archive for high school timetabling problems in different countries. Annals of Operations Research, v. 218, n. 1, p. 295-301, 2014.

Rodrigues, L. V. S., Casado, R. S. G. R., de Carvalho, E. N., Silva, M. M., e e Silva, L. C. Using FITradeoff in a ranking problem for supplier selection under TBL performance evaluation: An application in the textile sector. Production, v. 30, 2020.

Salo, A. A. e Hämäläinen, R. P. Preference assessment by imprecise ratio statements. Operations Research, v. 40, n. 6, p. 1053-1061, 1992.

Santos, H. G., Uchoa, E., Ochi, L. S., e Maculan, N. Strong bounds with cut and column generation for class-teacher timetabling. Annals of Operations Research, v. 194, n. 1, p. 399-412, 2012.

Saviniec, L., Santos, M. O., e Costa, A. M. Parallel local search algorithms for high school timetabling problems. European Journal of Operational Research, v. 265, n. 1, p. 81-98, 2018. 
Scala, N. M. e Pazour, J. A. A value model for asset tracking technology to support naval sea-based resupply. Engineering Management Journal, v. 28, n. 2, p. 120-130, 2016.

Sheng, H., Siau, K., e Nah, F. F.-H. Understanding the values of mobile technology in education: a value-focused thinking approach. Sigmis Database, v. 41, n. 2, p. 25-44, 2010.

Siebert, J. e Keeney, R. L. Creating more and better alternatives for decisions using objectives. Operations Research, v. 63, n. 5, p. 1144-1158, 2015.

Silva, M. M., de Gusmão, A. P. H., de Andrade, C. T. A., e Silva, W. The integration of VFT and FITradeoff multicriteria method for the selection of WCM projects. In: Anais da 2019 IEEE International Conference on Systems, Man and Cybernetics (SMC). Bari, Itália. IEEE, 2019. p. 1513-1517.

Sodenkamp, M. A., Tavana, M., e Di Caprio, D. Modeling synergies in multi-criteria supplier selection and order allocation: An application to commodity trading. European Journal of Operational Research, v. 254, n. 3, p. 859-874, 2016.

Sørensen, M. e Dahms, F. H. A two-stage decomposition of high school timetabling applied to cases in Denmark. Computers \& Operations Research, v. 43, p. 36-49, 2014.

Tan, J. S., Goh, S. L., Kendall, G., e Sabar, N. R. A survey of the state-of-the-art of optimisation methodologies in school timetabling problems. Expert Systems with Applications, v. 165, p. 113943, 2021.

Topcu, T. G. e Mesmer, B. L. Incorporating end-user models and associated uncertainties to investigate multiple stakeholder preferences in system design. Research in Engineering Design, v. 29, n. 3, p. 411-431, 2018.

Tuhkala, A., Isomäki, H., Hartikainen, M., Cristea, A. I., e Alessandrini, A. Identifying objectives for a learning space management system with value-focused thinking. In: Anais da the 9th International Conference on Computer Supported Education. Porto, Portugal. CSEDU, 2017. p. $25-34$.

Yuan, S., Li, T., e Wang, B. A co-evolutionary genetic algorithm for the two-machine flow shop group scheduling problem with job-related blocking and transportation times. Expert Systems with Applications, v. 152, p. 113360, 2020. 